\title{
Les lymphocytes B
}

Les lymphocytes B dérivent de la bourse de Fabricius chez les oiseaux ou de la moelle osseuse chez les mammifères (B comme " bursa " ou comme "bone marrow "). Les lymphocytes $\mathrm{B}$, qui représentent environ 5 à $15 \%$ des cellules lymphoïdes circulantes, sont caractérisés par la présence d'immunoglobulines à leur surface. Ces molécules sont ancrées dans la membrane et jouent le rôle de récepteur spécifique pour les antigènes. Les immunoglobulines de surface retrouvées sur les cellules lymphocytaires B périphériques humaines sont de deux types : IgM et $\operatorname{IgD}$ présents simultanément. En revanche, très peu de cellules expriment les IgG, les $\operatorname{Ig} \mathrm{A}$ ou les $\operatorname{IgE}$ à leur surface. Les lymphocytes B ont aussi des récepteurs pour les immunoglobulines (récepteurs $\mathrm{Fc}$ ), quileur permettent de former des rosettes EA, et des récepteurs pour le complément (CR1 ou récepteur de C3b, CR2 ou récepteur de C3d), leur permettant de former des rosettes EAC (voir Lexique $\mathrm{m} / \mathrm{s}^{\circ} 3$, vol. 3, p. 168).

Maturation des lymphocytes B

Durant la maturation des lymphocytes $B$, les gènes des immunoglobulines se réarrangent afin de créer la diversité de la cavité anticorps. Ce réarrangement n'est pas simultané pour les différentes chaînes ; il commence par la chaîne lourde, puis se poursuit par la chaîne légère kappa et enfin par la chaîne légère lambda. Dès qu'une immunoglobuline est produite (une chaîne lourde et une chaîne légère dont les gènes sont réarrangés et exprimés), les réarrangements des autres gènes semblent être stoppés. Les produits des gènes réarrangés sont également exprimés de façon asynchrone : la chaîne lourde mu apparaît la première dans le cytoplasme, puis les immunoglobulines complètes de surface sont synthétisées.

D'autres molécules, notamment des marqueurs de membrane, sont exprimées progressivement ou transitoirement. Tout ceci permet de classer les lymphocytes B en différentes catégories selon leur stade de maturation (Tableau I), ce qui est utile notamment pour classer les états malins (lymphomes et leucémies).

Tableau I

CARACTÉRISTIQUES DES LYMPHOCYTES B AUX DIFFÉRENTES ÉTAPES DE LEUR MATURATION

\begin{tabular}{|c|c|c|c|c|c|c|}
\hline \multirow[b]{2}{*}{$\begin{array}{l}\text { Réarrangement } \\
\text { des chaînes des } \\
\text { immunoglobulines }\end{array}$} & $\begin{array}{l}\text { Cellule } \\
\text { souche }\end{array}$ & Pré-B & B au repos & B activé & plasmocyte & \\
\hline & & & & & & $\begin{array}{l}\mu \\
\varkappa \\
\lambda\end{array}$ \\
\hline $\begin{array}{l}\text { Produit des gènes } \\
\text { des } \\
\text { immunoglobulines }\end{array}$ & & & & & & $\begin{array}{l}\text { Intracytoplasmique } \mu \\
\text { Immunoglobuline de surface }\end{array}$ \\
\hline Marqueurs & & & & & & $\begin{array}{l}\text { HLA DR }(29,33 \mathrm{kD}) \\
\text { CD } 19(95 \mathrm{kD})=\mathrm{B} 4 \\
\text { CD } 24(45,55,65 \mathrm{kD}) \\
\text { CD } 20(35 \mathrm{kD})=\mathrm{B} 1 \\
\text { CD } 10=\mathrm{CALLA}(100 \mathrm{kD}) \\
\text { CD } 37(40-45 \mathrm{kD}) \\
\text { CD } 22(135 \mathrm{kD}) \text { d'abord } \\
\text { intracytoplasmique puis de surface } \\
\text { CD } 21(40 \mathrm{kD})=\text { récepteur } \\
\text { pour EBV et pour C3d } \\
\text { (CR2) = B2 } \\
\text { CD } 35(220 \mathrm{kD})=\text { récep- } \\
\text { teur pour C3b (CR } 1)\end{array}$ \\
\hline
\end{tabular}




\section{Le rôle biologique}

Les lymphocytes B ont pour principal rôle de fabriquer les anticorps. La création de la diversité des anticorps par les réarrangements géniques, le passage d'une IgM à une autre classe d'immunoglobulines tout en conservant la même cavité anticorps, représentent des caractéristiques du lymphocyte B. Le passage d'une immunoglobuline ancrée à la membrane à une immunoglobuline sécrétée se fait par maturation différentielle des ARN messagers (épissage différentiel). Un mềme clone de lymphocytes $\mathrm{B}$ fabrique donc un seul type de cavité anticorps (idiotype) mais peut, durant les différentes étapes de l'immunisation, accoupler cet idiotype à des classes différentes d'immunoglobulines. Un clone de lymphocytes B est donc défini par l'idiotype de l'anticorps fabriqué, ou encore par le type de réarrangement génique correspondant à la partie variable (cavité anticorps) de l'immunoglobuline.

Laurent Degos

\section{BRÈVES}

Le TGF $\beta$ (transforming growth factor $\beta$ ) est la substance antiproliférative responsable de 1'effet cytostatique des antiœstrogènes sur les cancers du sein œstrogéno-dépendants. La lignée MCF 7, dérivée d'un cancer du sein sensible aux œstrogènes et ayant conservé ces propriétés, cesse de proliférer lorsqu'elle est traitée par des anti-œstrogènes. Ceux-ci induisent la maturation d'une forme inactive de TGF $\beta$ synthétisée par les cellules tumorales en une forme active qui inhibe leurs divisions (voir $\mathrm{m} / \mathrm{s}$ $n^{\circ} 8$, vol. 2, p. 467). Les cellules tumorales qui sont sensibles aux facteurs de croissance (par exemple le TGF $\alpha$ ) qu'elles sécrètent elles-mêmes (fonctionnement autocrine) peuvent également, par consequent, être soumises à un contrôle autocrine négatif.

[Knabbe C et al. Cell 1987; 48 : 417-20].

$m / s n^{\circ} 5$ ool. 3, mai 87

\section{BRÈVES}

- La résistance de certaines espèces de Plasmodium à la chloroquine serait liée à une anomalie de la répartition intracellulaire de la drogue, et non à l'absence de sa captation par les parasites : des souches sensibles de Plasmodium berghei concentrent la chloroquine dans la vacuole parasitaire alors que des souches résistantes la dispersent dans le cytoplasme. On ne sait pas encore si un tel phénomène est retrouvé pour Plasmodium falciparum, l'agent de la forme la plus grave de paludisme. Si tel était le cas, on pourrait envisager de coupler la chloroquine à des drogues cytotoxiques qui seraient ainsi "ciblées" vers les parasites.

[Moreau S et al. Eur J Cell Biol 1986 ; 42 : 207-10]

La phospholipase $\mathbf{A}_{2}$ (enzyme hydrolysant les phospholipides en acide gras et "lyso-phospholipide") du segment externe des bâtonnets participerait à la transduction visuelle. Elle serait couplée à deux G-protéines, la transducine et une espèce encore indéterminée. La transducine couplerait l'activation de la phospholipase $\mathrm{A}_{2}$ à la photolyse de la rhodopsine lors de l'illumination (voir m/s $n^{\circ} 4$, vol. 3, p. 192), alors que l'autre G-protéine contrôlerait négativement l'enzyme, assurant un rétrocontrôle. Le phénomène de transduction visuelle offre donc un exemple supplémentaire (nouvelle $\mathrm{m} / \mathrm{s}^{\circ} 4$, vol. $3, p$. 232) d'une $\mathrm{G}$-protéine couplée à deux systèmes effecteurs, la phosphodiestérase à GMPc via la sous-unité $\alpha$ et la phospholipase $A_{2}$ via, peutêtre, les sous-unités $\beta \gamma$. Il s'agit aussi d'un exemple d'un même système effecteur couplé à deux $\mathrm{G}$ protéines, l'une activatrice et l'autre inhibitrice... mais cela est déjà " banal "

[Jelsema Cl. I Biol Chem 1987 ; 262 : 163-8.]
Un canal ionique peut être ouvert, fermé... ou entrouvert! Différents acides aminés excitateurs (L-glutamate, Laspartate), jouant un rôle de neuromédiateurs, activent l'ouverture de canaux perméables au sodium $\left(\mathrm{Na}^{+}\right)$et au potassium $\left(\mathrm{K}^{+}\right)$, mais dont la perméabilité au calcium $\left(\mathrm{Ca}^{++}\right)$dépend de l'acide aminé étudié. On pensait donc jusqu'à présent que plusieurs systèmes de récepteurs étaient couplés à des canaux différents. Or, des résultats très importants qui viennent d'être publiés $[1,2]$ indiquent que les différentes molécules activatrices agissent, via leurs récepteurs et des Gprotéines, sur un même type de canal dont "l'état ", c'est-à-dire le degré d'ouverture, conditionne la perméabilité soit seulement au $\mathrm{Na}^{+}$et au $\mathrm{K}^{+}$. (état " entrouvert "), soit également au $\mathrm{Ca}^{++}$ (état " grand ouvert ").

1. Jahr CE, Stevenus CF. Nature $1987 ; 325$ : 522-5.]

[2. Cull-Candy SG, Usowicz MM. Nature 1987 ; 325 : 525-8.]

Les plaquettes semblent être des éléments essentiels des défenses antiparasitaires et les intermédiaires de l'effet thérapeutique de certaines drogues sur des parasites tels les microfilaires. Les filarioses à Loa-loa sont ainsi sensibles in vivo à une drogue, la diéthylcarbamazine qui, in vitro, est sans effet sur les microfilaires. En revanche, les plaquettes de malades traités tuent très rapidement les microfilaires... et aussi d'autres parasites, tels des bilharzies mélangées aux microfilaires. Il semble que les filaires libèrent un antigène qui stimule la cytotoxicité antiparasitaire des plaquettes de sujets recevant de la diéthylcarbamazine. Des radicaux libres produits par les plaquettes activées seraient responsables de l'effet antiparasitaire.

[Cesbron JY et al. Nature 1987 ; 325 : 533-6.] 